\title{
Interactive Multiobjective Optimization Using a Set of Additive Value Functions
}

\author{
José Rui Figueira ${ }^{1}$, Salvatore Greco ${ }^{2}$, Vincent Mousseau ${ }^{3}$, \\ and Roman Słowiński ${ }^{4,5}$ \\ 1 CEG-IST, Center for Management Studies, Instituto Superior Técnico, \\ Technical University of Lisbon, Portugal, figueira@ist.utl.pt \\ 2 Faculty of Economics, University of Catania, Corso Italia, 55, \\ 95129 Catania, Italy, salgreco@unict.it \\ 3 LAMSADE, Université Paris-Dauphine, 75775 Paris, France, \\ mousseau@lamsade.dauphine.fr \\ 4 Institute of Computing Science, Poznań University of Technology, \\ 60-965 Poznań, Poland, roman.slowinski@cs.put.poznan.pl \\ 5 Systems Research Institute, Polish Academy of Sciences, 01-447 Warsaw, Poland
}

\begin{abstract}
In this chapter, we present a new interactive procedure for multiobjective optimization, which is based on the use of a set of value functions as a preference model built by an ordinal regression method. The procedure is composed of two alternating stages. In the first stage, a representative sample of solutions from the Pareto optimal set (or from its approximation) is generated. In the second stage, the Decision Maker (DM) is asked to make pairwise comparisons of some solutions from the generated sample. Besides pairwise comparisons, the DM may compare selected pairs from the viewpoint of the intensity of preference, both comprehensively and with respect to a single criterion. This preference information is used to build a preference model composed of all general additive value functions compatible with the obtained information. The set of compatible value functions is then applied on the whole Pareto optimal set, which results in possible and necessary rankings of Pareto optimal solutions. These rankings are used to select a new sample of solutions, which is presented to the DM, and the procedure cycles until a satisfactory solution is selected from the sample or the DM comes to conclusion that there is no satisfactory solution for the current problem setting. Construction of the set of compatible value functions is done using ordinal regression methods called UTA ${ }^{\text {GMS }}$ and GRIP. These two methods generalize UTA-like methods and they are competitive to AHP and MACBETH methods. The interactive procedure will be illustrated through an example.
\end{abstract}

Reviewed by: Jerzy Błaszczyński, Poznań University of Technology, Poland Daisuke Sasaki, University of Cambridge, UK

Kalyanmoy Deb, Indian Institute of Technology Kanpur, India

J. Branke et al. (Eds.): Multiobjective Optimization, LNCS 5252, pp. 97-119, 2008.

(C) Springer-Verlag Berlin Heidelberg 2008 


\subsection{Introduction}

Over the last decade, research on MultiObjective Optimization (MOO) has been mainly devoted to generation of exact Pareto optimal solutions, or of an approximation of the Pareto optimal set (also called Pareto Frontier - PF), for problems with both combinatorial and multiple criteria structure. Only little attention has been paid to the inclusion of Decision Maker's (DM's) preferences in the generation process. MOO has been thus considered merely from the point of view of mathematical programming, while limited work is devoted to the point of view of decision aiding (see, however, Chapter 6 and Chapter 7, where preference information is used in evolutionary multiobjective optimization). There is no doubt, that the research about the inclusion of preferences within MOO is not sufficient, and thus the link between MOO and decision aiding should be strengthened. With this aim, in this chapter we propose to use the ordinal regression paradigm as a theoretically sound foundation for handling preference information in an interactive process of solving MOO problems.

In the following, we assume that the interactive procedure explores the PF of an MOO problem, however, it could be as well an approximation of this set.

The ordinal regression paradigm has been originally applied to multiple criteria decision aiding in the UTA method (Jacquet-Lagrèze and Siskos, 1982). This paradigm assumes construction of a criteria aggregation model compatible with preference information elicited from the DM. In the context of MOO, this information has the form of holistic judgments on a reference subset of the PF. The criteria aggregation model built in this way, is a DM's preference model. It is applied on the whole PF to show how the PF solutions compare between them using this model. The ordinal regression paradigm, gives a new sense to the interaction with the DM. The preference information is collected in a very easy way and concerns a small subset of PF solutions playing the role of a training sample. Elicitation of holistic pairwise comparisons of some solutions from the training sample, as well as comparisons of the intensity of preferences between some selected pairs of solutions, require from the DM a relatively small cognitive effort. The ordinal regression paradigm is also appropriate for designing an interactive process of solving a MOO problem, as a constructive learning process. This allows a DM to learn progressively about his/her preferences and make revisions of his/her judgments in successive iterations.

Designing an interactive process in a constructive learning perspective is based on the hypothesis that beyond the model definition, one of the prominent roles of the interactive process is to build a conviction in the mind of the DM on how solutions compare between them. Elaborating such a conviction is grounded on two aspects: (1) the preexisting elements, such as the DM's value system, past experience related to the decision problem; and (2) the elements of information presented to the DM in the dialogue stage, showing how the preference information from the previous iterations induces compar- 
isons of PF solutions. In order to be more specific about the nature of the constructive learning of preferences, it is important to say that there is a clear feedback in the process. On one hand, the preference information provided by the DM contributes to the construction of a preference model and, on the other hand, the use of the preference model shapes the DM's preferences or, at least, makes the DM's conviction evolve.

An interactive MOO procedure using the ordinal regression has been proposed in (Jacquet-Lagrèze et al., 1987). The ordinal regression implemented in this procedure is the same as in the UTA method, thus the preference model being used is a single additive value function with piecewise-linear components.

The interactive procedure proposed in this chapter is also based on ordinal regression, however, it is quite different from the previous proposal because it is using a preference model being a set of value functions, as considered in $\mathrm{UTA}^{\text {GMS }}$ and GRIP methods (Greco et al., 2003, 2008; Figueira et al., 2008). The value functions have a general additive form and they are compatible with preference information composed of pairwise comparisons of some solutions, and comparisons of the intensities of preference between pairs of selected solutions. UTA ${ }^{\text {GMS }}$ and GRIP methods extend the original UTA method in several ways: (1) all additive value functions compatible with the preference information are taken into account, while UTA is using only one such a function; (2) the marginal value functions are general monotone non-decreasing functions, and not only piecewise-linear ones, as in UTA. Moreover, the DM can provide a preference information which can be less demanding than in UTA (partial preorder of some solutions instead of a complete preorder), on one hand, and richer than in UTA (comparison of intensities of preference between selected pairs of solutions, e.g., the preference of $a$ over $b$ is stronger than the one of $c$ over $b$ ), on the other hand. Lastly, these methods provide as results necessary rankings that express statements that hold for all compatible value functions, and possible rankings that express statements which hold for at least one compatible value function, respectively. The two extensions of the UTA method appear to be very useful for organizing an interactive search of the most satisfactory solution of a MOO problem - the interaction with the DM is organized such that the preference information is provided incrementally, with the possibility of checking the impact of particular pieces of information on the preference structure of the PF.

The chapter is organized as follows. Section 4.2 is devoted to presentation of the general scheme of the constructive learning interactive procedure. Section 4.3 provides a brief reminder on learning of one compatible additive piecewise-linear value function for multiple criteria ranking problems using the UTA method. In Section 4.4, the GRIP method is presented, which is currently the most general of all UTA-like methods. GRIP is also competitive to the current main methods in the field of multiple criteria decision aiding. In particular, it is competitive to the AHP method (Saaty, 1980), which requires pairwise comparisons of solutions and criteria, and yields a priority ranking 
of solutions. Then, GRIP is competitive to the MACBETH method (Bana e Costa and Vansnick, 1994), which also takes into account a preference order of solutions and intensity of preference for pairs of solutions. The preference information used in GRIP does not need, however, to be complete: the DM is asked to provide comparisons of only those pairs of selected solutions on particular criteria for which his/her judgment is sufficiently certain. This is an important advantage when comparing GRIP to methods which, instead, require comparison of all possible pairs of solutions on all the considered criteria. Section 4.5 presents an application of the proposed interactive procedure for MOO; the possible pieces of preference information that can be considered in an interactive protocol are the following: ordinal pairwise comparisons of selected PF solutions, and ordinal comparisons of intensities of preference between selected pairs of PF solutions. In the last Section, some conclusions and further research directions are provided.

\subsection{Application of an Ordinal Regression Method within a Multiobjective Interactive Procedure}

In the following, we assume that the Pareto optimal set of a MOO problem is generated prior to an interactive exploration of this set. Instead of the whole and exact Pareto optimal set of a MOO problem, one can also consider a proper representation of this set, or its approximation. In any case, an interactive exploration of this set should lead the DM to a conviction that either there is no satisfactory solution to the considered problem, or there is at least one such a solution. We will focus our attention on the interactive exploration, and the proposed interactive procedure will be valid for any finite set of solutions to be explored. Let us denote this set by $A$. Note that such set $A$ can be computed using a MOO or EMO algorithm (see Chapters 1 and 3 ).

In the course of the interactive procedure, the preference information provided by the DM concerns a small subset of $A$, called reference or training sample, and denoted by $A^{R}$. The preference information is transformed by an ordinal regression method into a DM's preference model. We propose to use at this stage the GRIP method, thus the preference model is a set of general additive value functions compatible with the preference information. A compatible value function compares the solutions from the reference sample in the same way as the DM. The obtained preference model is then applied on the whole set $A$, which results in necessary and possible rankings of solutions. These rankings are used to select a new sample of reference solutions, which is presented to the DM, and the procedure cycles until a satisfactory solution is selected from the sample or the DM comes to conclusion that there is no satisfactory solution for the current problem setting.

The proposed interactive procedure is composed of the following steps: 
Step 1. Select a representative reference sample $A^{R}$ of solutions from set $A$.

Step 2. Present the sample $A^{R}$ to the DM.

Step 3. If the DM is satisfied with at least one solution from the sample, then this is the most preferred solution and the procedure stops. The procedure also stops in this step if, after several iterations, the DM concludes that there is no satisfactory solution for the current problem setting. Otherwise continue.

Step 4. Ask the DM to provide information about his/her preferences on set $A^{R}$ in the following terms:

- pairwise comparison of some solutions from $A^{R}$,

- comparison of intensities of comprehensive preferences between some pairs of solutions from $A^{R}$,

- comparison of intensities of preferences on single criteria between some pairs of solutions from $A^{R}$.

Step 5. Use the GRIP method to build a set of general additive value functions compatible with the preference information obtained from the DM in Step 4.

Step 6. Apply the set of compatible value functions built in Step 5 on the whole set $A$, and present the necessary and possible rankings (see sub-section 4.4.2) resulting from this application to the DM.

Step 7. Taking into account the necessary and possible rankings on set $A$, let the DM select a new reference sample of solutions $A^{R} \subseteq A$, and go to Step 2.

In Step 4, the information provided by the DM may lead to a set of constraints which define an empty polyhedron of the compatible value functions. In this case, the DM is informed what items of his/her preference information make the polyhedron empty, so as to enable revision in the next round. This point is explained in detail in (Greco et al., 2008; Figueira et al., 2008). Moreover, information provided by the DM in Step 4 cannot be considered as irreversible. Indeed, the DM can come back to one of previous iterations and continue from this point. This feature is concordant with the spirit of a learning oriented conception of multiobjective interactive optimization, i.e. it confirms the idea that the interactive procedure permits the DM to learn about his/her preferences and about the "shape" of the Pareto optimal set (see Chapter 15).

Notice that the proposed approach allows to elicit incrementally preference information by the DM. Remark that in Step 7, the "new" reference sample $A^{R}$ is not necessarily different from the previously considered, however, the preference information elicited by the DM in the next iteration is richer than previously, due to the learning effect. This permits to build and refine progressively the preference model: in fact, each new item of information provided 
in Step 4 reduces the set of compatible value functions and defines the DM's preferences more and more precisely.

Let us also observe that information obtained from the DM in Step 4 and information given to the DM in Step 6 is composed of very simple and easy to understand statements: preference comparisons in Step 4, and necessary and possible rankings in Step 6 (i.e., a necessary ranking that holds for all compatible value functions, and a possible ranking that holds for at least one compatible value function, see sub-section 4.4.2). Thus, the nature of information exchanged with the DM during the interaction is purely ordinal. Indeed, monotonically increasing transformations of evaluation scales of considered criteria have no influence on the final result.

Finally, observe that a very important characteristic of our method from the point of view of learning is that the DM can observe the impact of information provided in Step 4 in terms of necessary and possible rankings of solutions from set $A$.

\subsection{The Ordinal Regression Method for Learning One Compatible Additive Piecewise-Linear Value Function}

The preference information may be either direct or indirect, depending whether it specifies directly values of some parameters used in the preference model (e.g. trade-off weights, aspiration levels, discrimination thresholds, etc.) or, whether it specifies some examples of holistic judgments from which compatible values of the preference model parameters are induced. Eliciting direct preference information from the DM can be counterproductive in real-world decision making situations because of a high cognitive effort required. Consequently, asking directly the DM to provide values for the parameters seems to make the DM uncomfortable. Eliciting indirect preference is less demanding of the cognitive effort. Indirect preference information is mainly used in the ordinal regression paradigm. According to this paradigm, a holistic preference information on a subset of some reference or training solutions is known first and then a preference model compatible with the information is built and applied to the whole set of solutions in order to rank them.

The ordinal regression paradigm emphasizes the discovery of intentions as an interpretation of actions rather than as a priori position, which was called by March the posterior rationality (March, 1978). It has been known for at least fifty years in the field of multidimensional analysis. It is also concordant with the induction principle used in machine learning. This paradigm has been applied within the two main Multiple Criteria Decision Aiding (MCDA) approaches mentioned above: those using a value function as preference model (Srinivasan and Shocker, 1973; Pekelman and Sen, 1974; Jacquet-Lagrèze and Siskos, 1982; Siskos et al., 2005), and those using an outranking relation as preference model (Kiss et al., 1994; Mousseau and Slowinski, 1998). This 
paradigm has also been used since mid nineties' in MCDA methods involving a new, third family of preference models - a set of dominance decision rules induced from rough approximations of holistic preference relations (Greco et al., 1999, 2001, 2005; Słowiński et al., 2005).

Recently, the ordinal regression paradigm has been revisited with the aim of considering the whole set of value functions compatible with the preference information provided by the DM, instead of a single compatible value function used in UTA-like methods (Jacquet-Lagrèze and Siskos, 1982; Siskos et al., 2005). This extension has been implemented in a method called UTA GMS (Greco et al., 2003, 2008), further generalized in another method called GRIP (Figueira et al., 2008). UTA ${ }^{\text {GMS }}$ and GRIP are not revealing to the DM one compatible value function, but they are using the whole set of compatible (general, not piecewise-linear only) additive value functions to set up a necessary weak preference relation and a possible weak preference relation in the whole set of considered solutions.

\subsubsection{Concepts: Definitions and Notation}

We are considering a multiple criteria decision problem where a finite set of solutions $A=\{x, \ldots, y, \ldots w, \ldots\}$ is evaluated on a family $F=\left\{g_{1}, g_{2}, \ldots, g_{n}\right\}$ of $n$ criteria. Let $I=\{1,2, \ldots, n\}$ denote the set of criteria indices. We assume, without loss of generality, that the greater $g_{i}(x)$, the better solution $x$ on criterion $g_{i}$, for all $i \in I, x \in A$. A DM is willing to rank the solutions of $A$ from the best to the worst, according to his/her preferences. The ranking can be complete or partial, depending on the preference information provided by the DM and on the way of exploiting this information. The family of criteria $F$ is supposed to satisfy consistency conditions, i.e. completeness (all relevant criteria are considered), monotonicity (the better the evaluation of a solution on the considered criteria, the more it is preferable to another), and nonredundancy (no superfluous criteria are considered), see (Roy and Bouyssou, 1993).

Such a decision-making problem statement is called multiple criteria ranking problem. It is known that the only information coming out from the formulation of this problem is the dominance ranking. Let us recall that in the dominance ranking, solution $x \in A$ is preferred to solution $y \in A$ if and only if $g_{i}(x) \geq g_{i}(y)$ for all $i \in I$, with at least one strict inequality. Moreover, $x$ is indifferent to $y$ if and only if $g_{i}(x)=g_{i}(y)$ for all $i \in I$. Hence, for any pair of solutions $x, y \in A$, one of the four situations may arise in the dominance ranking: $x$ is preferred to $y, y$ is preferred to $x, x$ is indifferent to $y$, or $x$ is incomparable to $y$. Usually, the dominance ranking is very poor, i.e. the most frequent situation is: $x$ incomparable to $y$.

In order to enrich the dominance ranking, the DM has to provide preference information which is used to construct an aggregation model making the solutions more comparable. Such an aggregation model is called preference 
model. It induces a preference structure on set $A$, whose proper exploitation permits to work out a ranking proposed to the DM.

In what follows, the evaluation of each solution $x \in A$ on each criterion $g_{i} \in F$ will be denoted either by $g_{i}(x)$ or $x_{i}$.

Let $G_{i}$ denote the value set (scale) of criterion $g_{i}, i \in I$. Consequently,

$$
G=\prod_{i=1}^{n} G_{i}
$$

represents the evaluation space, and $x \in G$ denotes a profile of a solution in such a space. We consider a weak preference relation $\succsim$ on $A$ which means, for each pair of solutions $x, y \in A$,

$$
x \succsim y \Leftrightarrow \text { " } x \text { is at least as good as } y " .
$$

This weak preference relation can be decomposed into its asymmetric and symmetric parts, as follows,

1) $x \succ y \equiv[x \succsim y$ and $\operatorname{not}(y \succsim x)] \Leftrightarrow$ " $x$ is preferred to $y$ ", and

2) $x \sim y \equiv[x \succsim y$ and $y \succsim x] \Leftrightarrow$ " $x$ is indifferent to $y$ ".

From a pragmatic point of view, it is reasonable to assume that $G_{i} \subseteq \mathbb{R}$, for $i=1, \ldots, n$. More specifically, we will assume that the evaluation scale on each criterion $g_{i}$ is bounded, such that $G_{i}=\left[\alpha_{i}, \beta_{i}\right]$, where $\alpha_{i}, \beta_{i}, \alpha_{i}<\beta_{i}$ are the worst and the best (finite) evaluations, respectively. Thus, $g_{i}: A \rightarrow G_{i}$, $i \in I$. Therefore, each solution $x \in A$ is associated with an evaluation solution denoted by $\underline{g}(x)=\left(x_{1}, x_{2}, \ldots, x_{n}\right) \in G$.

\subsubsection{The UTA Method for a Multiple Criteria Ranking Problem}

In this sub-section, we recall the principle of the ordinal regression via linear programming, as proposed in the original UTA method, see (Jacquet-Lagrèze and Siskos, 1982).

\section{Preference Information}

The preference information is given in the form of a complete preorder on a subset of reference solutions $A^{R} \subseteq A$ (where $\left|A^{R}\right|=p$ ), called reference preorder. The reference solutions are usually those contained in set $A$ for which the DM is able to express holistic preferences. Let $A^{R}=\{a, b, c, \ldots\}$ be the set of reference solutions. 


\section{An Additive Model}

The additive value function is defined on $A$ such that for each $\underline{g}(x) \in G$,

$$
U(\underline{g}(x))=\sum_{i=1}^{n} u_{i}\left(g_{i}\left(x_{i}\right)\right),
$$

where, $u_{i}$ are non-decreasing marginal value functions, $u_{i}: G_{i} \rightarrow \mathbb{R}, i \in I$. For the sake of simplicity, we shall write (4.1) as follows,

$$
U(x)=\sum_{i=1}^{n} u_{i}\left(x_{i}\right) .
$$

In the UTA method, the marginal value functions $u_{i}$ are assumed to be piecewise-linear functions. The ranges $\left[\alpha_{i}, \beta_{i}\right]$ are divided into $\gamma_{i} \geq 1$ equal sub-intervals,

$$
\left[x_{i}^{0}, x_{i}^{1}\right],\left[x_{i}^{1}, x_{i}^{2}\right], \ldots,\left[x_{i}^{\gamma_{i}-1}, x_{i}^{\gamma_{i}}\right]
$$

where,

$$
x_{i}^{j}=\alpha_{i}+\frac{j}{\gamma_{i}}\left(\beta_{i}-\alpha_{i}\right), \quad j=0, \ldots, \gamma_{i}, \text { and } i \in I .
$$

The marginal value of a solution $x \in A$ is obtained by linear interpolation,

$$
u_{i}(x)=u_{i}\left(x_{i}^{j}\right)+\frac{x_{i}-x_{i}^{j}}{x_{i}^{j+1}-x_{i}^{j}}\left(u_{i}\left(x_{i}^{j+1}\right)-u_{i}\left(x_{i}^{j}\right)\right), \text { for } x_{i} \in\left[x_{i}^{j}, x_{i}^{j+1}\right] .
$$

The piecewise-linear additive model is completely defined by the marginal values at the breakpoints, i.e. $u_{i}\left(x_{i}^{0}\right)=u_{i}\left(\alpha_{i}\right), u_{i}\left(x_{i}^{1}\right), u_{i}\left(x_{i}^{2}\right), \ldots, u_{i}\left(x_{i}^{\gamma_{i}}\right)=$ $u_{i}\left(\beta_{i}\right)$.

In what follows, the principle of the UTA method is described as it was recently presented by Siskos et al. (2005).

Therefore, a value function $U(x)=\sum_{i=1}^{n} u_{i}\left(x_{i}\right)$ is compatible if it satisfies the following set of constraints

$$
\begin{aligned}
& \left.\begin{array}{l}
U(a)>U(b) \Leftrightarrow a \succ b \\
U(a)=U(b) \Leftrightarrow a \sim b
\end{array}\right\} \quad \forall a, b \in A^{R} \\
& u_{i}\left(x_{i}^{j+1}\right)-u_{i}\left(x_{i}^{j}\right) \geq 0, i=1, \ldots, n, j=1, \ldots, \gamma_{i}-1 \\
& u_{i}\left(\alpha_{i}\right)=0, i=1, \ldots, n \\
& \sum_{i=1}^{n} u_{i}\left(\beta_{i}\right)=1
\end{aligned}
$$

\section{Checking for Compatible Value Functions through Linear Programming}

To verify if a compatible value function $U(x)=\sum_{i=1}^{n} u_{i}\left(x_{i}\right)$ restoring the reference preorder $\succsim$ on $A^{R}$ exists, one can solve the following linear programming problem, where $u_{i}\left(x_{i}^{j}\right), i=1, \ldots, n, j=1, \ldots, \gamma_{i}$, are unknown, and $\sigma^{+}(a), \sigma^{-}(a)\left(a \in A^{R}\right)$ are auxiliary variables: 


$$
\left.\begin{array}{cl}
\text { Min } \rightarrow & F=\sum_{a \in A^{R}}\left(\sigma^{+}(a)+\sigma^{-}(a)\right) \\
\text { s.t. } & \\
& U(a)+\sigma^{+}(a)-\sigma^{-}(a) \geq \\
& U(b)+\sigma^{+}(b)-\sigma^{-}(b)+\varepsilon \Leftrightarrow a \succ b \\
& U(a)+\sigma^{+}(a)-\sigma^{-}(a)= \\
& U(b)+\sigma^{+}(b)-\sigma^{-}(b) \Leftrightarrow a \sim b
\end{array}\right\} \forall a, b \in A^{R}
$$

where, $\varepsilon$ is an arbitrarily small positive value so that $U(a)+\sigma^{+}(a)-\sigma^{-}(a)>$ $U(b)+\sigma^{+}(b)-\sigma^{-}(b)$ in case of $a \succ b$.

If the optimal value of the objective function of program (4.5) is equal to zero $\left(F^{*}=0\right)$, then there exists at least one value function $U(x)=\sum_{i=1}^{n} u_{i}\left(x_{i}\right)$ satisfying (4.4), i.e. compatible with the reference preorder on $A^{R}$. In other words, this means that the corresponding polyhedron (4.4) of feasible solutions for $u_{i}\left(x_{i}^{j}\right), i=1, \ldots, n, j=1, \ldots, \gamma_{i}$, is not empty.

Let us remark that the transition from the preorder $\succsim$ to the marginal value function exploits the ordinal character of the criterion scale $G_{i}$. Note, however, that the scale of the marginal value function is a conjoint interval scale. More precisely, for the considered additive value function $U(x)=\sum_{i=1}^{n} u_{i}\left(x_{i}\right)$, the admissible transformations on the marginal value functions $u_{i}\left(x_{i}\right)$ have the form $u_{i}^{*}\left(x_{i}\right)=k \times u_{i}\left(x_{i}\right)+h_{i}, h_{i} \in \mathbb{R}, i=1, \ldots, n, k>0$, such that for all $\left[x_{1}, \ldots, x_{n}\right],\left[y_{1}, \ldots, y_{n}\right] \in G$

$$
\sum_{i=1}^{n} u_{i}\left(x_{i}\right) \geq \sum_{i=1}^{n} u_{i}\left(y_{i}\right) \Leftrightarrow \sum_{i=1}^{n} u_{i}^{*}\left(x_{i}\right) \geq \sum_{i=1}^{n} u_{i}^{*}\left(y_{i}\right) .
$$

An alternative way of representing the same preference model is:

$$
U(x)=\sum_{i=1}^{n} w_{i} \hat{u}_{i}(x),
$$

where $\hat{u}\left(\alpha_{i}\right)=0, \hat{u}\left(\beta_{i}\right)=1, w_{i} \geq 0 i=1,2, \ldots, n$ and $\sum_{i=1}^{n} w_{i}=1$. Note that the correspondence between (4.6) and (4.2) is such that $w_{i}=u_{i}\left(\beta_{i}\right), \forall i \in$ $G$. Due to the cardinal character of the marginal value function scale, the parameters $w_{i}$ can be interpreted as tradeoff weights among marginal value functions $\hat{u}_{i}(x)$. We will use, however, the preference model (4.2) with normalization constraints bounding $U(x)$ to the interval $[0,1]$.

When the optimal value of the objective function of the program (4.5) is greater than zero $\left(F^{*}>0\right)$, then there is no value function $U(x)=\sum_{i=1}^{n} u_{i}\left(x_{i}\right)$ compatible with the reference preorder on $A^{R}$. In such a case, three possible moves can be considered: 
- increasing the number of linear pieces $\gamma_{i}$ for one or several marginal value function $u_{i}$ could make it possible to find an additive value function compatible with the reference preorder on $A^{R}$;

- revising the reference preorder on $A^{R}$ could lead to find an additive value function compatible with the new preorder;

- searching over the relaxed domain $F \leq F^{*}+\eta$ could lead to an additive value function giving a preorder on $A^{R}$ sufficiently close to the reference preorder (in the sense of Kendall's $\tau$ ).

\subsection{The Ordinal Regression Method for Learning the Whole Set of Compatible Value Functions}

Recently, two new methods, UTA ${ }^{\text {GMS }}$ (Greco et al., 2008) and GRIP (Figueira et al., 2008), have generalized the ordinal regression approach of the UTA method in several aspects:

- taking into account all additive value functions (4.1) compatible with the preference information, while UTA is using only one such function,

- considering marginal value functions of (4.1) as general non-decreasing functions, and not piecewise-linear, as in UTA,

- asking the DM for a ranking of reference solutions which is not necessarily complete (just pairwise comparisons),

- taking into account additional preference information about intensity of preference, expressed both comprehensively and with respect to a single criterion,

- avoiding the use of the exogenous, and not neutral for the result, parameter $\varepsilon$ in the modeling of strict preference between solutions.

$\mathrm{UTA}^{\text {GMS }}$ produces two rankings on the set of solutions $A$, such that for any pair of solutions $a, b \in A$ :

- in the necessary ranking, $a$ is ranked at least as good as $b$ if and only if, $U(a) \geq U(b)$ for all value functions compatible with the preference information,

- in the possible ranking, $a$ is ranked at least as good as $b$ if and only if, $U(a) \geq U(b)$ for at least one value function compatible with the preference information.

GRIP produces four more necessary and possible rankings on the set of solutions $A \times A$ as it can bee seen in sub-section 4.4.2.

The necessary ranking can be considered as robust with respect to the preference information. Such robustness of the necessary ranking refers to the fact that any pair of solutions compares in the same way whatever the additive value function compatible with the preference information. Indeed, when no preference information is given, the necessary ranking boils down to the weak 
dominance relation (i.e., $a$ is necessarily at least as good as $b$, if $g_{i}(a) \geq g_{i}(b)$ for all $g_{i} \in F$ ), and the possible ranking is a complete relation. Every new pairwise comparison of reference solutions, for which the dominance relation does not hold, is enriching the necessary ranking and it is impoverishing the possible ranking, so that they converge with the growth of the preference information.

Moreover, such an approach has another feature which is very appealing in the context of MOO. It stems from the fact that it gives space for interactivity with the DM. Presentation of the necessary ranking, resulting from a preference information provided by the DM, is a good support for generating reactions from part of the DM. Namely, (s)he could wish to enrich the ranking or to contradict a part of it. Such a reaction can be integrated in the preference information considered in the next calculation stage.

The idea of considering the whole set of compatible value functions was originally introduced in UTA ${ }^{\text {GMS }}$. GRIP (Generalized Regression with Intensities of Preference) can be seen as an extension of UTA ${ }^{\text {GMS }}$ permitting to take into account additional preference information in form of comparisons of intensities of preference between some pairs of reference solutions. For solutions $x, y, w, z \in A$, these comparisons are expressed in two possible ways (not exclusive): $(i)$ comprehensively, on all criteria, like " $x$ is preferred to $y$ at least as much as $w$ is preferred to $z$ "; and, (ii) partially, on each criterion, like " $x$ is preferred to $y$ at least as much as $w$ is preferred to $z$, on criterion $g_{i} \in F$ ". Although UTA ${ }^{\text {GMS }}$ was historically the first method among the two, as GRIP incorporates and extends UTA ${ }^{\text {GMS }}$, in the following we shall present only GRIP.

\subsubsection{The Preference Information Provided by the Decision Maker}

The DM is expected to provide the following preference information in the dialogue stage of the procedure:

- A partial preorder $\succsim$ on $A^{R}$ whose meaning is: for some $x, y \in A^{R}$

$$
x \succsim y \Leftrightarrow \text { " } x \text { is at least as good as } y " .
$$

Moreover, $\succ$ (preference) is the asymmetric part of $\succsim$, and $\sim$ (indifference) is its symmetric part.

- A partial preorder $\succsim^{*}$ on $A^{R} \times A^{R}$, whose meaning is: for some $x, y, w$, $z \in A^{R}$,

$(x, y) \succsim^{*}(w, z) \Leftrightarrow$ " $x$ is preferred to $y$ at least as much as $w$ is preferred to $z^{\prime \prime}$.

Also in this case, $\succ^{*}$ is the asymmetric part of $\succsim^{*}$, and $\sim^{*}$ is its symmetric part.

- A partial preorder $\succsim_{i}^{*}$ on $A^{R} \times A^{R}$, whose meaning is: for some $x, y, w, z \in$ $A^{R},(x, y) \succsim_{i}^{*}(w, z) \Leftrightarrow$ " $x$ is preferred to $y$ at least as much as $w$ is preferred to $z$ " on criterion $g_{i}, i \in I$. 
In the following, we also consider the weak preference relation $\succsim_{i}$ being a complete preorder whose meaning is: for all $x, y \in A$,

$$
x \succsim_{i} y \Leftrightarrow \quad \text { " } x \text { is at least as good as } y \text { " on criterion } g_{i}, \quad i \in I \text {. }
$$

Weak preference relations $\succsim_{i}, i \in I$, are not provided by the DM, but they are obtained directly from the evaluation of solutions $x$ and $y$ on criteria $g_{i}$, i.e., $x \succsim_{i} y \Leftrightarrow g_{i}(x) \geq g_{i}(y), i \in I$.

\subsubsection{Necessary and Possible Binary Relations in Set $A$ and in Set $\boldsymbol{A} \times \boldsymbol{A}$}

When there exists at least one value function compatible with the preference information provided by the DM, the method produces the following rankings:

- a necessary ranking $\succsim^{N}$, for all pairs of solutions $(x, y) \in A \times A$;

- a possible ranking $\succsim^{P}$, for all pairs of solutions $(x, y) \in A \times A$;

- a necessary ranking $\succsim^{*^{N}}$, with respect to the comprehensive intensities of preferences for all $((x, y),(w, z)) \in A \times A \times A \times A$;

- a possible ranking $\succsim^{*^{P}}$, with respect to the comprehensive intensities of preferences for all $((x, y),(w, z)) \in A \times A \times A \times A$;

- a necessary ranking $\succsim_{i}^{*^{N}}$, with respect to the partial intensities of preferences for all $((x, y),(w, z)) \in A \times A \times A \times A$ and for all criteria $g_{i}$, $i \in I$;

- a possible ranking $\succsim_{i}^{*^{P}}$, with respect to the partial intensities of preferences for all $((x, y),(w, z)) \in A \times A \times A \times A$ and for all criteria $g_{i}, i \in I$.

\subsubsection{Linear Programming Constraints}

In this sub-section, we present a set of constraints that interprets the preference information in terms of conditions on the compatible value functions.

To be compatible with the provided preference information, the value function $U: A \rightarrow[0,1]$ should satisfy the following constraints corresponding to DM's preference information:

a) $U(w)>U(z)$ if $w \succ z$

b) $U(w)=U(z)$ if $w \sim z$

c) $U(w)-U(z)>U(x)-U(y)$ if $(w, z) \succ^{*}(x, y)$

d) $U(w)-U(z)=U(x)-U(y)$ if $(w, z) \sim^{*}(x, y)$

e) $u_{i}(w) \geq u_{i}(z)$ if $w \succsim_{i} z, i \in I$

f) $u_{i}(w)-u_{i}(z)>u_{i}(x)-u_{i}(y)$ if $(w, z) \succ_{i}^{*}(x, y), i \in I$

g) $u_{i}(w)-u_{i}(z)=u_{i}(x)-u_{i}(y)$ if $(w, z) \sim_{i}^{*}(x, y), i \in I$

Let us remark that within UTA-like methods, constraint $a$ ) is written as $U(w) \geq U(z)+\varepsilon$, where $\varepsilon>0$ is a threshold exogenously introduced. Analogously, constraints $c$ ) and $f$ ) should be written as, 


$$
U(w)-U(z) \geq U(x)-U(y)+\varepsilon
$$

and

$$
u_{i}(w)-u_{i}(z) \geq u_{i}(x)-u_{i}(y)+\varepsilon .
$$

However, we would like to avoid the use of any exogenous parameter and, therefore, instead of setting an arbitrary value of $\varepsilon$, we consider it as an auxiliary variable, and we test the feasibility of constraints $a$ ), $c$ ), and $f$ ) (see sub-section 4.4.4). This permits to take into account all possible value functions, even those having a very small preference threshold $\varepsilon$. This is also safer from the viewpoint of "objectivity" of the used methodology. In fact, the value of $\varepsilon$ is not meaningful in itself and it is useful only because it permits to discriminate preference from indifference.

Moreover, the following normalization constraints should also be taken into account:

h) $u_{i}\left(x_{i}^{*}\right)=0$, where $x_{i}^{*}$ is such that $x_{i}^{*}=\min \left\{g_{i}(x): x \in A\right\}$;

i) $\sum_{i \in I} u_{i}\left(y_{i}^{*}\right)=1$, where $y_{i}^{*}$ is such that $y_{i}^{*}=\max \left\{g_{i}(x): x \in A\right\}$.

\subsubsection{Computational Issues}

In order to conclude the truth or falsity of binary relations $\succsim^{N}, \succsim^{P}, \succsim^{*^{N}}$, $\succsim^{*^{P}}, \succsim_{i}^{*^{N}}$ and $\succsim_{i}^{*^{P}}$, we have to take into account that, for all $x, y, w, z \in A$ and $i \in I$ :

1) $x \succsim^{N} y \Leftrightarrow \inf \{U(x)-U(y)\} \geq 0$,

2) $x \succsim^{P} y \Leftrightarrow \inf \{U(y)-U(x)\} \leq 0$,

3) $(x, y) \succsim^{*^{N}}(w, z) \Leftrightarrow \inf \{(U(x)-U(y))-(U(w)-U(z))\} \geq 0$,

4) $(x, y) \succsim *^{P}(w, z) \Leftrightarrow \inf \{(U(w)-U(z))-(U(x)-U(y))\} \leq 0$,

5) $(x, y) \succsim_{i}^{*^{N}}(w, z) \Leftrightarrow \inf \left\{\left(u_{i}\left(x_{i}\right)-u_{i}\left(y_{i}\right)\right)-\left(u_{i}\left(w_{i}\right)-u_{i}\left(z_{i}\right)\right)\right\} \geq 0$,

6) $(x, y) \succsim_{i}^{*^{P}}(w, z) \Leftrightarrow \inf \left\{\left(u_{i}\left(w_{i}\right)-u_{i}\left(z_{i}\right)\right)-\left(u_{i}\left(x_{i}\right)-u_{i}\left(y_{i}\right)\right)\right\} \leq 0$,

with the infimum calculated on the set of value functions satisfying constraints from $a$ ) to $i$ ). Let us remark, however, that the linear programming is not able to handle strict inequalities such as the above $a$ ), $c$ ), and $f$ ). Moreover, linear programming permits to calculate the minimum or the maximum of an objective function and not an infimum. Nevertheless, reformulating properly the above properties 1) to 6), a result presented in (Marichal and Roubens, 2000) permits to use linear programming for testing the truth of binary relations, $\succsim^{N}, \succsim^{P}, \succsim^{*^{N}}, \succsim^{*^{P}}, \succsim_{i}^{*^{N}}$ and $\succsim_{i}^{*^{P}}$.

In order to use such a result, constraints $a$ ), c) and $f$ ) have to be reformulated as follows: 
$\left.a^{\prime}\right) U(x) \geq U(y)+\varepsilon$ if $x \succ y$;

$\left.c^{\prime}\right) U(x)-U(y) \geq U(w)-U(z)+\varepsilon$ if $(x, y) \succ^{*}(w, z)$;

$\left.f^{\prime}\right) u_{i}(x)-u_{i}(y) \geq u_{i}(w)-u_{i}(z)+\varepsilon$ if $(x, y) \succ_{i}^{*}(w, z)$.

Notice that constraints $a$ ), c) and $f$ ) are equivalent to $\left.a^{\prime}\right), c^{\prime}$ ), and $f^{\prime}$ ) whenever $\varepsilon>0$.

After properties 1) -6) have to be reformulated such that the search of the infimum is replaced by the calculation of the maximum value of $\varepsilon$ on the set of value functions satisfying constraints from $a$ ) to $i$ ), with constraints $a$ ), $c$ ), and $f$ ) transformed to $\left.a^{\prime}\right), c^{\prime}$ ), and $f^{\prime}$ ), plus constraints specific for each point:

$\left.1^{\prime}\right) x \succsim^{P} y \Leftrightarrow \varepsilon^{*}>0$,

where $\varepsilon^{*}=\max \varepsilon$, subject to the constraints $\left.\left.\left.\left.\left.a^{\prime}\right), b\right), c^{\prime}\right), d\right), e\right), f^{\prime}$ ), plus the constraint $U(x) \geq U(y)$;

$\left.2^{\prime}\right) x \succsim^{N} y \Leftrightarrow \varepsilon^{*} \leq 0$,

where $\varepsilon^{*}=\max \varepsilon$, subject to the constraints $\left.\left.\left.\left.\left.a^{\prime}\right), b\right), c^{\prime}\right), d\right), e\right), f^{\prime}$ ), plus the constraint $U(y) \geq U(x)+\varepsilon$;

$\left.3^{\prime}\right)(x, y) \succsim^{*^{P}}(w, z) \Leftrightarrow \varepsilon^{*}>0$,

where $\varepsilon^{*}=\max \varepsilon$, subject to the constraints $\left.\left.\left.\left.\left.\left.a^{\prime}\right), b\right), c^{\prime}\right), d\right), e\right), f^{\prime}\right)$, plus the constraint $((U(x)-U(y))-(U(w)-U(z))) \geq 0$;

$\left.4^{\prime}\right)(x, y) \succsim *^{N}(w, z) \Leftrightarrow \varepsilon^{*} \leq 0$, where $\varepsilon^{*}=\max \varepsilon$, subject to the constraints $\left.\left.\left.\left.\left.a^{\prime}\right), b\right), c^{\prime}\right), d\right), e\right), f^{\prime}$ ), plus the constraint $((U(w)-U(z))-(U(x)-U(y))) \geq \varepsilon$;

$\left.5^{\prime}\right)(x, y) \succsim_{i}^{*^{P}}(w, z) \Leftrightarrow \varepsilon^{*}>0$,

where $\varepsilon^{*}=\max \varepsilon$, subject to the constraints $\left.\left.\left.\left.\left.a^{\prime}\right), b\right), c^{\prime}\right), d\right), e\right), f^{\prime}$ ), plus the constraint $\left(u_{i}\left(x_{i}\right)-u_{i}\left(y_{i}\right)\right)-\left(u_{i}\left(w_{i}\right)-u_{i}\left(z_{i}\right)\right) \geq 0$;

$\left.6^{\prime}\right)(x, y) \succsim_{i}^{*^{N}}(w, z) \Leftrightarrow \varepsilon^{*} \leq 0$,

where $\varepsilon^{*}=\max \varepsilon$, subject to the constraints $\left.\left.\left.\left.\left.\left.a^{\prime}\right), b\right), c^{\prime}\right), d\right), e\right), f^{\prime}\right)$, plus the constraint $\left(\left(u_{i}\left(w_{i}\right)-u_{i}\left(z_{i}\right)\right)-\left(u_{i}\left(x_{i}\right)-u_{i}\left(y_{i}\right)\right) \geq \varepsilon\right.$.

\subsubsection{Comparison of GRIP with the Analytical Hierarchy Process}

In AHP (Saaty, 1980, 2005), criteria should be compared pairwise with respect to their importance. Actions (solutions) are also compared pairwise on particular criteria with respect to intensity of preference. The following nine point scale of preference is used: 1 - equal importance, 3 - moderate importance, 5 - strong importance, 7 - very strong or demonstrated importance, and 9 - extreme importance. 2, 4, 6 and 8 are intermediate values between the two adjacent judgements. The intensity of importance of criterion $g_{i}$ over criterion $g_{j}$ is the inverse of the intensity of importance of $g_{j}$ over $g_{i}$. Analogously, the intensity of preference of action $x$ over action $y$ is the inverse of the intensity 
of preference of $y$ over $x$. The above scale is a ratio scale. Therefore, the intensity of importance is read as the ratio of weights $w_{i}$ and $w_{j}$ corresponding to criteria $g_{i}$ and $g_{j}$, and the intensity of preference is read as the ratio of the attractiveness of $x$ and the attractiveness of $y$, with respect to the considered criterion $g_{i}$. In terms of value functions, the intensity of preference can be interpreted as the ratio $\frac{u_{i}\left(g_{i}(x)\right)}{u_{i}\left(g_{i}(y)\right)}$. Thus, the problem is how to obtain values of $w_{i}$ and $w_{j}$ from ratio $\frac{w_{i}}{w_{j}}$, and values of $u_{i}\left(g_{i}(x)\right)$ and $u_{i}\left(g_{i}(y)\right)$ from ratio $\frac{u_{i}\left(g_{i}(x)\right)}{u_{i}\left(g_{i}(y)\right)}$.

In AHP, it is proposed that these values are supplied by principal eigenvectors of matrices composed of the ratios $\frac{w_{i}}{w_{j}}$ and $\frac{u_{i}\left(g_{i}(x)\right)}{u_{i}\left(g_{i}(y)\right)}$. The marginal value functions $u_{i}\left(g_{i}(x)\right)$ are then aggregated by means of a weighted-sum using the weights $w_{i}$.

Comparing AHP with GRIP, we can say that with respect to a single criterion, the type of questions addressed to the DM is the same: express the intensity of preference in qualitative-ordinal terms (equal, moderate, strong, very strong, extreme). However, differently from GRIP, this intensity of preference is translated into quantitative terms (the scale from 1 to 9 ) in a quite arbitrary way. In GRIP, instead, the marginal value functions are just a numerical representation of the original qualitative-ordinal information, and no intermediate transformation into quantitative terms is exogenously imposed.

Other differences between AHP and GRIP are related to the following aspects.

1) In GRIP, the value functions $u_{i}\left(g_{i}(x)\right)$ depend mainly on holistic judgements, i.e. comprehensive preferences involving jointly all the criteria, while this is not the case in AHP.

2) In AHP, the weights $w_{i}$ of criteria $g_{i}$ are calculated on the basis of pairwise comparisons of criteria with respect to their importance; in GRIP, this is not the case, because the value functions $u_{i}\left(g_{i}(x)\right)$ are expressed on the same scale and thus they can be summed up without any further weighting.

3) In AHP, all non-ordered pairs of actions must be compared from the viewpoint of the intensity of preference with respect to each particular criterion. Therefore, if $m$ is the number of actions, and $n$ the number of criteria, then the DM has to answer $n \times \frac{m \times(m-1)}{2}$ questions. Moreover, the DM has to answer questions relative to $\frac{n \times(n-1)}{2}$ pairwise comparisons of considered criteria with respect to their importance. This is not the case in GRIP, which accepts partial information about preferences in terms of pairwise comparison of some reference actions. Finally, in GRIP there is no question about comparison of relative importance of criteria.

As far as point 2) is concerned, observe that the weights $w_{i}$ used in AHP represent tradeoffs between evaluations on different criteria. For this reason it is doubtful if they could be inferred from answers to questions concerning comparison of importance. Therefore, AHP has a problem with meaningfulness of its output with respect to its input, and this is not the case of GRIP. 


\subsubsection{Comparison of GRIP with MACBETH}

MACBETH (Measuring Attractiveness by a Categorical Based Evaluation TecHnique) (Bana e Costa and Vansnick, 1994; Bana e Costa et al., 2005) is a method for multiple criteria decision analysis that appeared in the early nineties. This approach requires from the DM qualitative judgements about differences of value to quantify the relative attractiveness of actions (solutions) or criteria.

When using MACBETH, the DM is asked to provide preference information composed of a strict order of all actions from $A$, and a qualitative judgement of the difference of attractiveness between all two non-indifferent actions. Seven semantic categories of the difference of attractiveness are considered: null, very weak, weak, moderate, strong, very strong, and extreme. The difference of attractiveness reflects the intensity of preferences.

The main idea of MACBETH is to build an interval scale from the preference information provided by the DM. It is, however, necessary that the above categories correspond to disjoint intervals (represented in terms of the real numbers). The bounds for such intervals are not arbitrarily fixed a priori, but they are calculated so as to be compatible with the numerical values of all particular actions from $A$, and to ensure compatibility between these values (see Bana e Costa et al. 2005). Linear programming models are used for these calculations. In case of inconsistent judgments, MACBETH provides the DM with information in order to eliminate such inconsistency.

When comparing MACBETH with GRIP, the following aspects should be considered:

- both deal with qualitative judgements;

- both need a set of comparisons of actions or pairs of actions to work out a numerical representation of preferences, however, MACBETH depends on the specification of two characteristic levels on the original scale, "neutral" and "good", to obtain the numerical representation of preferences, while GRIP does not need this information;

- GRIP adopts the "disaggregation-aggregation" approach and, therefore, it considers mainly holistic judgements relative to comparisons involving jointly all the criteria, which is not the case of MACBETH;

- GRIP is more general than MACBETH since it can take into account the same kind of qualitative judgments as MACBETH (the difference of attractiveness between pairs of actions) and, moreover, the intensity of preferences of the type " $x$ is preferred to $y$ at least as much as $z$ is preferred to $w "$.

As for the last item, it should be noticed that the intensity of preference considered in MACBETH and the intensity coming from comparisons of the type " $x$ is preferred to $y$ at least as strongly as $w$ is preferred to $z$ " (i.e., the quaternary relation $\succsim^{*}$ ) are substantially the same. In fact, the intensities of preference are equivalence classes of the preorder generated by ${ }^{*}$. This means 
that all the pairs $(x, y)$ and $(w, z)$, such that $x$ is preferred to $y$ with the same intensity as $w$ is preferred to $z$, belong to the same semantic category of difference of attractiveness considered in MACBETH. To be more precise, the structure of intensity of preference considered in MACBETH is a particular case of the structure of intensity of preference represented by ${ }^{*}$ in GRIP. Still more precisely, GRIP has the same structure of intensity as MACBETH when $\succsim^{*}$ is a complete preorder. When this does not occur, MACBETH cannot be used while GRIP can naturally deal with this situation.

Comparison of GRIP and MACBETH could be summarized in the following points:

1. GRIP is using preference information relative to: 1) comprehensive preference on a subset of reference actions with respect to all criteria, 2) marginal intensity of preference on some single criteria, and 3) comprehensive intensity of preference with respect to all criteria, while MACBETH requires preference information on all pairs of actions with respect to each one of the considered criteria.

2. Information about marginal intensity of preference is of the same nature in GRIP and MACBETH (equivalence classes of relation $\succsim_{i}^{*}$ correspond to qualitative judgements of MACBETH), but in GRIP it may not be complete.

3. GRIP is a "disaggregation-aggregation" approach while MACBETH makes use of the "aggregation" approach and, therefore, it needs weights to aggregate evaluations on the criteria.

4. GRIP works with all compatible value functions, while MACBETH builds a single interval scale for each criterion, even if many such scales would be compatible with preference information.

5. Distinguishing necessary and possible consequences of using all value functions compatible with preference information, GRIP includes a kind of robustness analysis instead of using a single "best-fit" value function.

6. The necessary and possible preference relations considered in GRIP have several properties of general interest for MCDA.

\subsection{An Illustrative Example}

In this section, we illustrate how our approach can support the DM to specify his/her preferences on a set of Pareto optimal solutions. In this didactic example, we shall imagine an interaction with a fictitious DM so as to exemplify and illustrate the type of interaction proposed in our method.

We consider a MOO problem that involves five objectives that are to be maximized. Let us consider a subset $A$ of the Pareto Frontier of a MOO problem consisting of 20 solutions (see Table 4.1). Note that this set $A$ can be computed using a MOO or EMO algorithm (see Chapters 2 and 3). Let us suppose that the reference sample $A^{R}$ of solutions from set $A$ is the following: 
Table 4.1. The whole set of Pareto optimal solutions for the example MOO problem

\begin{tabular}{ll}
\hline$s_{1}=(14.5,147,4,1014,5.25)$ & $s_{11}=(15.75,164.375,41.5,311,6.5)$ \\
$s_{2}=(13.25,199.125,4,1014,4)$ & $s_{12}=(13.25,181.75,41.5,311,4)$ \\
$s_{3}=(15.75,164.375,16.5,838.25,5.25)$ & $s_{13}=(12,199.125,41.5,311,2.75)$ \\
$s_{4}=(12,181.75,16.5,838.25,4)$ & $s_{14}=(17,147,16.5,662.5,5.25)$ \\
$s_{5}=(12,164.375,54,838.25,4)$ & $s_{15}=(15.75,199.125,16.5,311,6.5)$ \\
$s_{6}=(13.25,199.125,29,662.5,5.25)$ & $s_{16}=(13.25,164.375,54,311,4)$ \\
$s_{7}=(13.25,147,41.5,662.5,5.25)$ & $s_{17}=(17,181.75,16.5,486.75,5.25)$ \\
$s_{8}=(17,216.5,16.5,486.75,1.5)$ & $s_{18}=(14.5,164.375,41.5,838.25,4)$ \\
$s_{9}=(17,147,41.5,486.75,5.25)$ & $s_{19}=(15.75,181.75,41.5,135.25,5.25)$ \\
$s_{10}=(15.75,216.5,41.5,662.5,1.5)$ & $s_{20}=(15.75,181.75,41.5,311,2.75)$ \\
\hline
\end{tabular}

$A^{R}=\left\{s_{1}, s_{2}, s_{4}, s_{5}, s_{8}, s_{10}\right\}$. For the sake of simplicity, we shall consider the set $A^{R}$ constant across iterations (although the interaction scheme permits $A^{R}$ to evolve during the process). For the same reason, we will suppose that the DM expresses preference information only in terms of pairwise comparisons of solutions from $A^{R}$ (intensity of preference will not be expressed in the preference information).

The DM does not see any satisfactory solution in the reference sample $A^{R}$ $\left(s_{1}, s_{2}, s_{4}\right.$ and $s_{5}$ have too weak evaluations on the first criterion, while $s_{8}$ and $s_{10}$ have the worst evaluation in $A$ on the last criterion), and wishes to find a satisfactory solution in $A$. Obviously, solutions in $A$ are not comparable unless preference information is expressed by the DM. In this perspective, he/she provides a first comparison: $s_{1} \succ s_{2}$.

Considering the provided preference information, we can compute the necessary and possible rankings on set $A$ (computation of this example were performed using the GNU-UTA software package (Chakhar and Mousseau, 2007); note that the UTA ${ }^{\text {GMS }}$ and GRIP methods are also implemented in the Decision Deck software platform (Consortium, 2008)). The DM decided to consider the necessary ranking only, as it has more readable graphical representation than the possible ranking at the stage of relatively poor preference information. The partial preorder of the necessary ranking is depicted in Fig. 4.1 and shows the comparisons that hold for all additive value functions compatible with the information provided by the DM (i.e., $s_{1} \succ s_{2}$ ). It should be observed that the computed partial preorder contains the preference information provided by the DM (dashed arrow), but also additional comparisons that result from the initial information (continuous arrows); for instance, $s_{3} \succ^{N} s_{4}$ holds because $U\left(s_{3}\right) \geq U\left(s_{4}\right)$ for each compatible value function (this gives $\left.s_{3} \succsim^{N} s_{4}\right)$ and $U\left(s_{3}\right)>U\left(s_{4}\right)$ for at least one value function (this gives $\left.\operatorname{not}\left(s_{4} \succsim^{N} s_{3}\right)\right)$.

Analyzing this first result, the DM observes that the necessary ranking is still very poor which makes it difficult to discriminate among the solutions in $A$. He/she reacts by stating that $s_{4}$ is preferred to $s_{5}$. Considering this new piece of preference information, the necessary ranking is computed again 


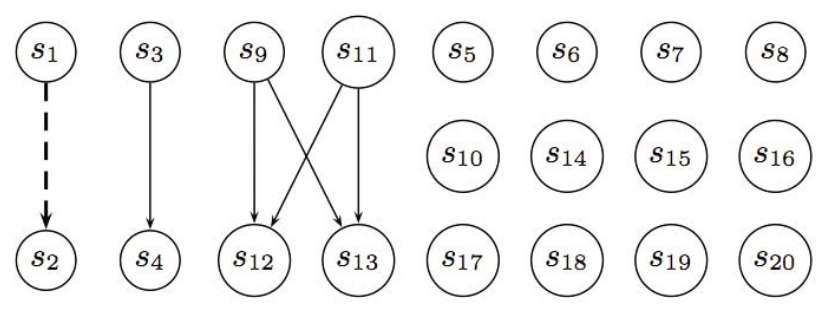

Fig. 4.1. Necessary partial ranking at the first iteration

and shown in Fig. 4.2. At this second iteration, it should be observed that the resulting necessary ranking has been enriched as compared to the first iteration (bold arrows), narrowing the set of "best choices", i.e., solutions that are not preferred by any other solution in the necessary ranking: $\left\{s_{1}, s_{3}, s_{6}\right.$, $\left.s_{8}, s_{10}, s_{14}, s_{15}, s_{17}, s_{18}, s_{19}, s_{20}\right\}$.

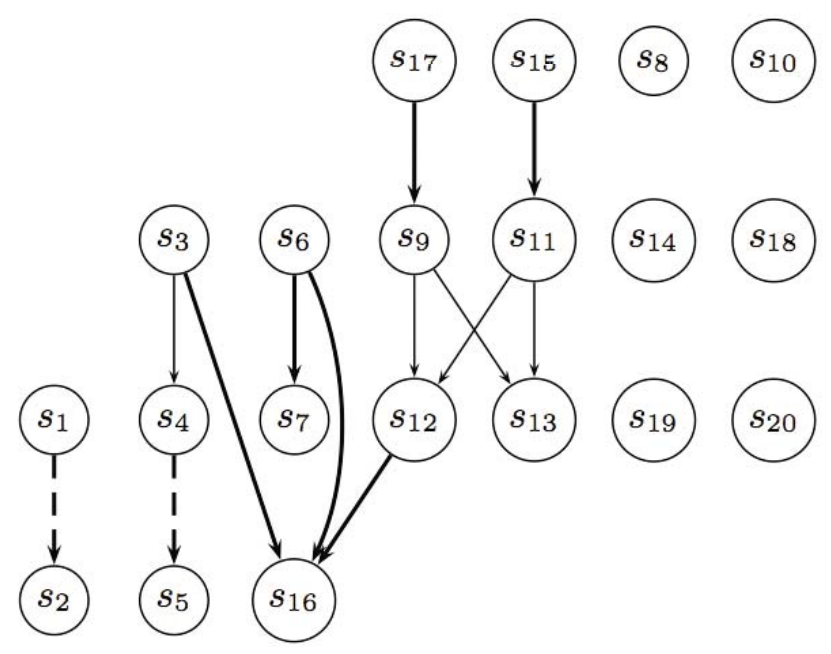

Fig. 4.2. Necessary partial ranking at the second iteration

The DM believes that this necessary ranking is still insufficiently decisive and adds a new comparison: $s_{8}$ is preferred to $s_{10}$. Once again, the necessary ranking is computed and shown in Fig. 4.3.

At this stage, the set of possible "best choices" has been narrowed down to a limited number of solutions, among which $s_{14}$ and $s_{17}$ are judged satisfactory by the DM. In fact, these two solutions have a very good performance on the first criterion without any "dramatic" evaluation on the other criteria. 


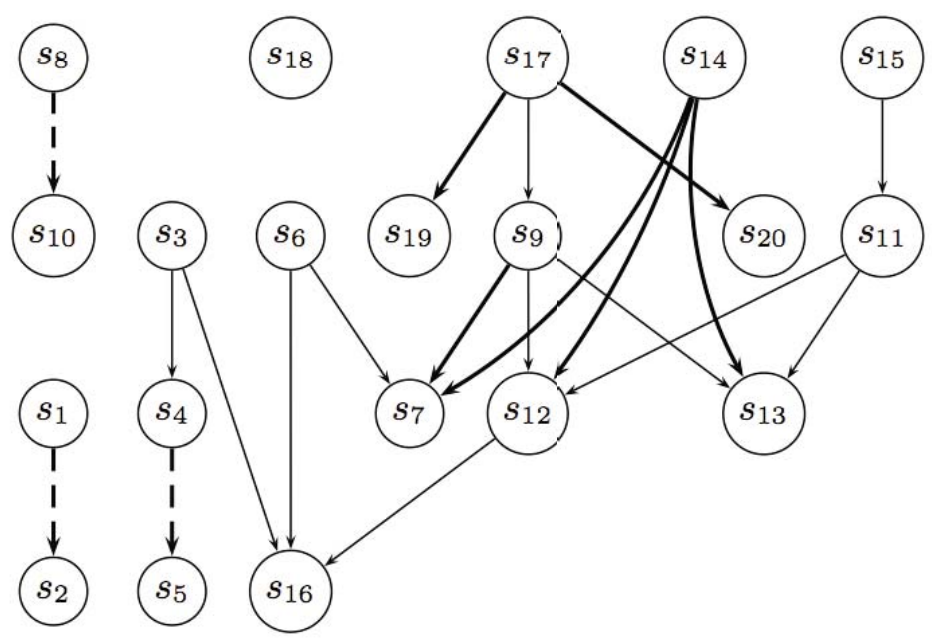

Fig. 4.3. Necessary partial ranking at the third iteration

The current example stops at this step, but the DM could then decide to provide further preference information to enrich the necessary ranking. He/she could also compute new Pareto optimal solutions "close" to $s_{14}$ and $s_{17}$ to zoom investigations in this area. In this example we have shown that the proposed interactive process supports the DM in choosing most satisfactory solutions, without imposing any strong cognitive effort, as the only information required is a holistic preference information.

\subsection{Conclusions and Further Research Directions}

In this chapter, we introduced a new interactive procedure for multiobjective optimization. It consists in an interactive exploration of a Pareto optimal set, or its approximation, generated prior to the exploration using a MOO or EMO algorithm. The procedure represents a constructive learning approach, because on one hand, the preference information provided by the DM contributes to the construction of a preference model and, on the other hand, the use of the preference model shapes the DM's preferences or, at least, makes DM's convictions evolve.

Contrary to many existing MCDA methods, the proposed procedure does not require any excessive cognitive effort from the DM because the preference information is of a holistic nature and, moreover, it can be partial. Due to distinguishing necessary and possible consequences of using all value functions compatible with the preference information, the procedure is also robust, comparing to methods using a single "best-fit" value function. This is a feature of uttermost importance in $\mathrm{MOO}$. 
An almost immediate extension of the procedure could consist in admitting preference information in form of a sorting of selected Pareto optimal solutions into some pre-defined and preference ordered classes. Providing such an information could be easier for some DMs than making the pairwise comparisons.

\section{Acknowledgements}

The first and the third authors acknowledge the support from Luso-French PESSOA bilateral cooperation. The fourth author wishes to acknowledge financial support from the Polish Ministry of Science and Higher Education. All authors acknowledge, moreover, the support of the COST Action IC0602 "Algorithmic Decision Theory".

\section{References}

Bana e Costa, C.A., Vansnick, J.C.: MACBETH: An interactive path towards the construction of cardinal value functions. International Transactions in Operational Research 1(4), 387-500 (1994)

Bana e Costa, C.A., De Corte, J.M., Vansnick, J.C.: On the mathematical foundation of MACBETH. In: Figueira, J., Greco, S., Ehrgott, M. (eds.) Multiple Criteria Decision Analysis: State of the Art Surveys, pp. 409-443. Springer Science + Business Media Inc., New York (2005)

Chakhar, S., Mousseau, V.: GNU-UTA: a GNU implementation of UTA methods (2007), http://www. lamsade.dauphine.fr/ mousseau/GNU-UTA

Consortium, D.D.: Decision deck: an open-source software platform for mcda methods (2006-2008), www.decision-deck.org

Figueira, J., Greco, S., Słowiński, R.: Building a set of additive value functions representing a reference preorder and intensities of preference: GRIP method. European Journal of Operational Research, to appear

Greco, S., Matarazzo, B., Słowiński, R.: The use of rough sets and fuzzy sets in MCDM. In: Gal, T., Hanne, T., Stewart, T. (eds.) Multicriteria Decision Making: Advances in MCDM Models, Algorithms, Theory and Applications, pp. 1-14. Kluwer Academic Publishers, Dordrecht (1999)

Greco, S., Matarazzo, B., Slowinski, R.: Rough sets theory for multicriteria decision analysis. European Journal of Operational Research 129, 1-47 (2001)

Greco, S., Mousseau, V., Słowiński, R.: Assessing a partial preorder of alternatives using ordinal regression and additive utility functions: A new UTA method. In: 58th Meeting of the EURO Working Group on MCDA, Moscow (2003)

Greco, S., Matarazzo, B., Słowiński, R.: Decision rule approach. In: Figueira, J., Greco, S., Ehrgott, M. (eds.) Multiple Criteria Decision Analysis: State of the Art Surveys, pp. 507-562. Springer Science + Business Media Inc., New York (2005)

Greco, S., Mousseau, V., Słowiński, R.: Ordinal regression revisited: Multiple criteria ranking with a set of additive value functions. European Journal of Operational Research 191(2), 416-436 (2008) 
Jacquet-Lagrèze, E., Siskos, Y.: Assessing a set of additive utility functions for multicriteria decision making: The UTA method. European Journal of Operational Research 10(2), 151-164 (1982)

Jacquet-Lagrèze, E., Meziani, R., Słowiński, R.: MOLP with an interactive assessment of a piecewise linear utility function. European Journal of Operational Research 31, 350-357 (1987)

Kiss, L., Martel, J., Nadeau, R.: ELECCALC - an interactive software for modelling the decision maker's preferences. Decision Support Systems 12(4-5), 757777 (1994)

March, J.: Bounded rationality, ambiguity and the engineering of choice. Bell Journal of Economics 9, 587-608 (1978)

Marichal, J., Roubens, M.: Determination of weights of interacting criteria from a reference set. European Journal of Operational Research 124(3), 641-650 (2000)

Mousseau, V., Slowinski, R.: Inferring an ELECTRE TRI model from assignment examples. Journal of Global Optimization 12(2), 157-174 (1998)

Pekelman, D., Sen, S.: Mathematical programming models for the determination of attribute weights. Management Science 20(8), 1217-1229 (1974)

Roy, B., Bouyssou, D.: Aide Multicritère à la Décision: Méthodes et Cas. Economica, Paris (1993)

Saaty, T.: The Analytic Hierarchy Process. McGraw Hill, New York (1980)

Saaty, T.: The analytic hierarchy and analytic network processes for the measurement of intangible criteria and for decision-making. In: Figueira, J., Greco, S., Ehrgott, M. (eds.) Multiple Criteria Decision Analysis: The State of the Art Surveys, pp. 345-407. Springer Science+Business Media, Inc., New York (2005)

Siskos, Y., Grigoroudis, V., Matsatsinis, N.: UTA methods. In: Figueira, F., Greco, S., Ehrgott, M. (eds.) Multiple Criteria Decision Analysis: State of the Art Surveys, pp. 297-343. Springer Science + Business Media Inc., New York (2005)

Słowiński, R., Greco, S., Matarazzo, B.: Rough set based decision support. In: Burke, E., Kendall, G. (eds.) Introductory Tutorials on Optimization, Search and Decision Support Methodologies, pp. 475-527. Springer Science + Business Media Inc., New York (2005)

Srinivasan, V., Shocker, A.: Estimating the weights for multiple attributes in a composite criterion using pairwise judgments. Psychometrika 38(4), 473-493 (1973) 